\title{
Expansion and State-Dependent Variability along Sensory Processing Streams
}

\begin{abstract}
DRenato Duarte
Institute of Neuroscience and Medicine (INM-6) and Institute for Advanced Simulation (IAS-6), Jülich Research Center and JARA, 52425 Jülich, Germany, Bernstein Center Freiburg and Faculty of Biology, Albert-Ludwig University of Freiburg, 79104 Freiburg im Breisgau, Germany, and Institute of Adaptive and Neural Computation, School of Informatics, University of Edinburgh, Edinburgh EH8 9LE, United Kingdom
\end{abstract}

Review of Schölvinck et al. (2015)

The fidelity with which cortical sensory areas represent environmental stimuli poses a strong constraint on the quality, suitability, and precision of any subsequent computation and, ultimately, on the organism's behavioral performance. Understanding the precise nature of such representations, however, is significantly complicated by the fact that natural stimuli do not simply drive cortical activity, but rather modulate it via complex interactions with the local circuit's ongoing activity. This state dependence of cortical responses to stimuli underlies a pervasive phenomenon observed in primary sensory systems: a high degree of variability in population responses to repeated presentations of well controlled stimuli (Arieli et al., 1996; Kisley and Gerstein, 1999), which, at first sight, appears at odds with the representational fidelity required for adequate processing and communication.

It is thus essential to gain a better insight into the mechanistic nature of such response variability, its origin, and its precise relation with cortical states. Given the spike timing precision and trial-to-trial reliability of single cortical neurons in re-

Received March 6, 2015; revised March 26, 2015; accepted April 2, 2015. The author declares no competing financial interests.

Correspondence should be addressed to Renato Duarte, Jülich Research Center and JARA, Institute of Neuroscience and Medicine (INM-6) and Institute for Advanced Simulation (IAS-6), Building 15.22, 52425 Jülich, Germany. E-mail: r.duarte@fz-juelich.de.

DOI:10.1523/JNEUROSCI.0874-15.2015

Copyright $\odot 2015$ the authors $\quad 0270-6474 / 15 / 357315-02 \$ 15.00 / 0$ sponse to noisy input currents (Mainen and Sejnowski, 1995), it is reasonable to assume that variability is not generated by intrinsic neuronal properties, but by the massive convergence of synaptic inputs onto those neurons, most of which are excitatory and capable of eliciting small, sub-millivolt fluctuations of the neuron's membrane potential. This assumption is reinforced by the observation of correlated variability in the responses of individual sensory cortical neurons, which is likely to reflect shared presynaptic sources and thus highlight the functional organization of the circuit.

To shed some light into the origins of variability and its relation to ongoing cortical states, Scholvinck et al. (2015) began by recording from lateral geniculate nucleus (LGN) and V1 neurons in the cat visual pathway and analyzing the different responses of single neurons when presented with the same visual stimulus under well-controlled conditions. The visual stimuli used for this purpose as well as for all subsequent analyses consisted of sequentially flashed stationary gratings, randomly sampled from a stimulus set comprising stimuli with different grating orientations and spatial phases, to probe the response specificity (or lack thereof) to the relevant stimulus features.

They found an increase in variability in V1 relative to LGN neurons (Scholvinck et al., 2015, their Fig. 1), which is consistent with previous accounts (Kara et al.,
2000; Carandini, 2004) and suggests that the source of cortical variability is not inherited from thalamic inputs. In fact, a previous study (Kara et al., 2000) demonstrated an inverse relationship between variability and firing density along the visual processing stream, whereby response variability almost doubled from peripheral sensory neurons [retinal ganglion cells (RGCs)] to LGN relay neurons and from LGN to V1, while simultaneously, the mean firing rates in each of these processing stages consistently decreased from retina to LGN to V1. This relationship can be partly explained as a result of a stepwise dimensionality expansion and consequent response sparsification along the involved populations. As the relevant representations are transferred through a random projection from a lower- to a higher-dimensional state space, the degrees of freedom available increase and the stimulus representation loses its uniqueness and trial-to-trial reproducibility. The distributed activity patterns used to represent the stimuli become much sparser in the expansion, which is an important feature if the structure of the signal needs to be recovered at later stages (Ganguli and Sompolinsky, 2012).

Such expansive transformations along the sensory streams thus involve the projection of compressed and dense stimulus representations onto less unique, but spatially segmented, high-dimensional sparse representations. The result is a greatly re- 
duced overlap between activity patterns elicited in response to different stimuli, thereby providing a significant computational advantage by simplifying the discrimination and categorization of sensory representations (Babadi and Sompolinsky, 2014).

However, the total amount of variability observed in cortical population responses cannot be fully accounted for by the sparsity of the expanded representation, and it is deeply interconnected with the characteristics of ongoing activity upon stimulus arrival. Whether the computational benefits of the expansion are fully exploited by the processing circuit depends on the current state of the circuit, which can, as demonstrated by Scholvinck et al. (2015) and further discussed below, have a dramatic impact on the reliability of stimulus-evoked responses.

The state of cortical circuits varies along a continuum characterized by modulations in the depth of spontaneous fluctuations of neural population activity (see, e.g., Harris and Thiele, 2011). On one extreme of this continuum, population activity is coordinated and dominated by strong lowfrequency fluctuations with a high, positive mean pairwise correlation in spiking activity. In this state, known as the synchronous state, strong population bursts are interspersed by periods of network silence (also referred to as up- and down-states). On the other extreme lies the asynchronous state characterized by smaller fluctuations in global firing rates, along with the corresponding suppression of low-frequency oscillations. In this state, pairwise correlations are very low, as is the average population firing rate.

These variations of global state are most prominent during different stages of the sleep cycle, when the whole cortex is engaged in a similar activity profile, but they also exist (albeit less prominently) in awake animals. The key distinguishing feature is that, in this case, different cortical areas engage in different active states, depending on current task processing demands, and the state transitions are more subtle. Typically, alert and actively behaving animals exhibit a highly desynchronized state in the engaged cortical areas [e.g., during active sensing, the mouse barrel cortex displays asynchronous activity (Poulet and Petersen, 2008)] while awake but quiescent animals display a more synchronized activity state with strong population-wide fluctuations. Studies exploring the representational ca- pacity of different cortical systems operating in these different regimes clearly demonstrate the advantages of active desynchronization for appropriate representation of temporally extended events (Marguet and Harris, 2011; Duarte and Morrison, 2014; Pachitariu et al., 2015), whereas synchronized states appear to respond mostly to discrete, isolated (pulselike) events (Otazu et al., 2009), which can be thought of as a mechanism of attentional control to activate the idle cortical circuit (Harris and Thiele, 2011).

To better understand the nature of response variability in $\mathrm{V} 1$ and its relation to cortical states, Scholvinck et al. (2015) assessed the degree to which variability is shared across multiple cortical sites and its relation to the structure of ongoing activity upon stimulus arrival. Their results show that much of the variability is indeed shared across the population and is mostly independent of orientation preference, reflecting global, population-wide fluctuations observed during synchronous states. These global fluctuations could adequately account for the observed pairwise correlations measured during spontaneous activity and during visual responses and were not significantly higher between recording sites corresponding to regions of similar orientation preference, which implies that the variations in cortical state, which largely determine the observed response variability, tend to engage the overall population regardless of the functional organization of the circuit. The amount of trial-to-trial variability of the responses observed was thus highly correlated with the size of population-wide fluctuations (Scholvinck et al., 2015, their Fig. 3), which further demonstrates the significantly diminished ability of cortical circuits to accurately represent their input when operating in a synchronous state.

It is worth noting that the different states and their transitions observed in anesthetized animals [which was the case in the studies performed by Scholvinck et al. (2015)] can be significantly different from those obtained under normal physiological conditions. Nevertheless, the most prominent features and dynamical properties of these states are retained and the careful use of anesthesia has been shown to provide greater control over cortical states without the added confounding effects present in awake conditions.

The observed differences in variability and correlation structure between different evoked responses appears to be mostly determined by the characteristics of ongoing activity and its spontaneous fluctuations. These results imply that temporally precise and reliable responses require an actively desynchronized circuit, which enforces the required sparsity of population activity and allows adequate stimulus representations to emerge as a spatiotemporal succession of network states, thus highlighting a fundamental computational principle of neocortical circuits.

\section{References}

Arieli A, Sterkin A, Grinvald A, Aertsen A (1996) Dynamics of ongoing activity: explanation of the large variability in evoked cortical responses. Science 273:1668-1671. CrossRef Medline

Babadi B, Sompolinsky H (2014) Sparseness and expansion in sensory representations. Neuron 83:1213-1226. CrossRef Medline

Carandini M (2004) Amplification of trial-totrial response variability by neurons in visual cortex. PLoS Biol 2:E264. CrossRef Medline

Duarte R, Morrison A (2014) Dynamic stability of sequential stimulus representations in adapting neuronal networks. Front Comp Neurosci 8:124. CrossRef Medline

Ganguli S, Sompolinsky H (2012) Compressed sensing, sparsity, and dimensionality in neuronal information processing and data analysis. Ann Rev Neurosci 35:485-508. CrossRef Medline

Harris KD, Thiele A (2011) Cortical state and attention. Nat Rev Neurosci 12:509-523. CrossRef Medline

Kara P, Reinagel P, Reid RC (2000) Low response variability in simultaneously recorded retinal, thalamic, and cortical neurons. Neuron 27:635-646. CrossRef Medline

Kisley MA, Gerstein GL (1999) Trial-to-trial variability and state-dependent modulation of auditory-evoked responses in cortex. J Neurosci 19:10451-10460. Medline

Mainen ZF, Sejnowski TJ (1995) Reliability of spike timing in neocortical neurons. Science 268:1503-1506. CrossRef Medline

Marguet SL, Harris KD (2011) State-dependent representation of amplitude-modulated noise stimuli in rat auditory cortex. J Neurosci 31: 6414-6420. CrossRef Medline

Otazu GH, Tai LH, Yang Y, Zador AM (2009) Engaging in an auditory task suppresses responses in auditory cortex. Nat Neurosci 12 : 646-654. CrossRef Medline

Pachitariu M, Lyamzin DR, Sahani M, Lesica NA (2015) State-dependent population coding in primary auditory cortex. J Neurosci 35 : 2058-2073. CrossRef Medline

Poulet JF, Petersen CC (2008) Internal brain state regulates membrane potential synchrony in barrel cortex of behaving mice. Nature 454: 881-885. CrossRef Medline

Schölvinck ML, Saleem AB, Benucci A, Harris KD, Carandini M (2015) Cortical state determines global variability and correlations in visual cortex. J Neurosci 35:170-178. CrossRef Medline 